\title{
Parameter $\theta$-Type Marcinkiewicz Integral on Nonhomogeneous Weighted Generalized Morrey Spaces
}

\author{
Guanghui Lu \\ College of Mathematics and Statistics, Northwest Normal University, Lanzhou, Gansu 730070, China \\ Correspondence should be addressed to Guanghui Lu; lghwmm1989@126.com
}

Received 24 July 2020; Revised 30 September 2020; Accepted 10 December 2020; Published 16 December 2020

Academic Editor: Yoshihiro Sawano

Copyright ( 2020 Guanghui Lu. This is an open access article distributed under the Creative Commons Attribution License, which permits unrestricted use, distribution, and reproduction in any medium, provided the original work is properly cited.

Let $(\mathscr{X}, d, \mu)$ be a nonhomogeneous metric measure space satisfying the upper doubling and geometrically doubling conditions in the sense of Hytönen. In this setting, the author proves that parameter $\theta$-type Marcinkiewicz integral $\mathscr{M}_{\theta}^{\rho}$ is bounded on the weighted generalized Morrey space $L^{p, \phi, \tau}(\omega)$ for $p \in(1, \infty)$. Furthermore, the boudedness of $\mathscr{M}_{\theta}^{\rho}$ on weak weighted generalized Morrey space $W L^{p, \phi, \tau}(\omega)$ is also obtained.

\section{Introduction}

To unify the spaces of homogeneous type in the sense of Coifman and Weiss (see $[1,2])$ and nondoubling measure spaces (see [3-8]), in 2010, Hytönen [9] first introduced a new class of metric measure space satisfying the so-called upper doubling and geometrically doubling conditions. For the sake of convenience, the new space is now called a nonhomogeneous metric measure space. Since then, the research on the space has been widely focused, for example, some authors established the properties of function spaces on the nonhomogeneous metric measure space (see [10-14]). On the other hand, the boundedness of singular integral operators on various of spaces is also obtained; the readers can see [15-20] and so on.

In this paper, let $(\mathscr{X}, d, \mu)$ be a nonhomogeneous metric measure space in the sense of Hytönen [9]. In this setting, we will give out the definition of weighted (weak) generalized Morrey space and then obtain the boundedness of parameter $\theta$-type Marcinkiewicz integral $\mathscr{M}_{\theta}^{\rho}$. In 1938, Morrey [21] first introduced the definition of Morrey space when regularity of the solution of elliptic differential equations in terms of the solutions themselves and their derivatives is considered. Later, many researchers studied Morrey spaces from various point of view. After studying Morrey spaces in detail, some researchers passed to generalized Morrey spaces, weighted
Morrey spaces, and generalized Morrey spaces, for example, the Guliyev, Mizuhara, and Nakai in [22-24] introduced generalized Morrey spaces $M_{p, \varphi}\left(\mathbb{R}^{n}\right)$ and also obtained some boundedness of integral operators on $M_{p, \varphi}\left(\mathbb{R}^{n}\right)$. In addition, we can see $[25,26]$ to study the research and development about generalized Morrey space and weak generalized Morrey space. In 2009, Komori and Shirai [27] defined the weighted Morrey space and studied the boundedness of some classical operators such as the Hardy-Littlewood maximal operator and Calderón-Zygmund operator on these spaces. Based on this, Nakamura and Sawano established the boundedness of singular integral operator and its commutator on weighted Morrey space (see [28]). In 2012, Guliyev [29] first introduced the generalized weighted Morrey spaces $M^{p, \rho}(\omega)$ and studied the boundedness of the sublinear operators and their higher order commutators which is generated by Calderón-Zygmund operators and Riese potentials on these spaces (see also [30, 31]). In 2016, Nakamura defined another definition of generalized weighted Morrey space and established the boundedness of classical operators on this space (see [32]).

Recently, the Morrey space, weighted Morrey space, and generalized Morrey space on $\mathbb{R}^{n}$ have been extended to nonhomogeneous metric measure space, for example, we can see $[10,13,14]$. Motivated by these, in this paper, we first give out the definition of weighted generalized Morrey space 
and weighted weak generalized Morrey space on $(\mathscr{X}, d, \mu)$. Also, we obtain the boundedness of parameter $\theta$-type Marcinkiewicz integral $\mathscr{M}_{\theta}^{\rho}$ on the weighted generalized Morrey space and weak generalized Morrey space.

Before stating the main results of this paper, we first recall some necessary notions. The following definitions of upper doubling condition and geometrically doubling condition are from [9].

Definition 1 [9]. A metric measure space $(\mathscr{X}, d, \mu)$ is said to be upper doubling if $\mu$ is a Borel measure on $\mathscr{X}$ and there exist a dominating function $\lambda: \mathscr{X} \times(0, \infty) \longrightarrow(0, \infty)$ and a constant $C_{(\lambda)}>0$, depending on $\lambda$, for each $x \in \mathscr{X}, r \longrightarrow \lambda$ $(x, r)$ is nondecreasing and, for all $x \in \mathscr{X}$ and $r \in(0, \infty)$

$$
\mu(B(x, r)) \leq \lambda(x, r) \leq C_{(\lambda)} \lambda\left(x, \frac{r}{2}\right) .
$$

Moreover, Hytönen et al. [12] have showed that there exists another dominating function $\tilde{\lambda}$ such that $\tilde{\lambda} \leq \lambda, C_{(\tilde{\lambda})}$ $\leq C_{(\lambda)}$, and for all $x, y \in \mathscr{X}$ with $d(x, y) \leq r$

$$
\tilde{\lambda}(x, r) \leq C_{(\tilde{\lambda})} \tilde{\lambda}(y, r) .
$$

If there is no special explanation in this paper, we always assume that $\lambda$ satisfies (2).

Definition 2 [9]. A metric space $(\mathscr{X}, d)$ is said to be geometrically doubling if there exist some $N_{0} \in \mathbb{N}$ such that, for any ball $B(x, r) \subset \mathscr{X}$ with $x \in \mathscr{X}$ and $r \in(0, \infty)$, there exists a finite ball covering $\left\{B\left(x_{i}, r / 2\right)\right\}_{i}$ of $B(x, r)$ such that the cardinality of this covering is at most $N_{0}$.

Remark 3. Let $(\mathscr{X}, d)$ be a metric measure space. Hytönen in [9] pointed out that the geometrically doubling $(\mathscr{X}, d)$ is equivalent to the following statement: for any $\varepsilon \in(0,1)$ and any ball $B(x, r) \subset \mathscr{X}$ with $x \in \mathscr{X}$ and $r \in(0, \infty)$, there exists a finite ball covering $\left\{B\left(x_{i}, \varepsilon r\right)\right\}_{i}$ of $B(x, r)$ such that the cardinality of this covering is at most $N_{0} \varepsilon^{-n_{0}}$, where $n_{0}:=\log _{2} N_{0}$

Although the measure doubling condition is not assumed uniformly for all balls in the nonhomogeneous metric measure space $(\mathscr{X}, d, \mu)$, Hytönen in [9] showed that there exist many balls which have the $(\alpha, \beta)$-doubling properties. That is, for all $\alpha, \beta \in(1, \infty)$, a ball $B \subset \mathscr{X}$ is said to be $(\alpha, \beta)$-doubling if $\mu(\alpha B) \leq \beta \mu(B)$. To be precise, Hytönen [9] pointed out that, if a metric measure space $(\mathscr{X}, d, \mu)$ is upper doubling and $\alpha, \beta \in(1, \infty)$ with $\beta>\left[C_{(\lambda)}\right]^{\log _{2} \alpha}=: \alpha^{\nu}$, then there exists some $j \in \mathbb{Z}_{+}$such that $\alpha^{j} B$ is $(\alpha, \beta)$-doubling. Moreover, let $(\mathscr{X}, d)$ be a geometrically doubling, $\beta>\alpha^{n_{0}}$ and $\mu$ be a Borel measure on $\mathscr{X}$ being finite on bounded sets. Hytönen also showed that, for $\mu$-a.e. $x \in \mathscr{X}$, there exist arbitrary small $(\alpha, \beta)$-doubling balls with centers at $x$. Furthermore, the radii of these balls may be chosen to be of the form $\alpha^{-j} r$ for $j \in \mathbb{N}$ and any preassigned number $r \in(0, \infty)$. Throughout this paper, for any $\alpha \in(1, \infty)$ and ball $B$, the smallest
( $\left.\alpha, \beta_{\alpha}\right)$-doubling ball of the form $\alpha^{j} B$ with $j \in \mathbb{Z}_{+}$is simply denoted by $\tilde{B}^{\alpha}$, where

$$
\beta_{\alpha}:=\alpha^{3\left(\max \left\{n_{0}, \mu\right\}\right)}+[\max \{5 \alpha, 30\}]^{n_{0}}+[\max \{3 \alpha, 30\}]^{\nu} .
$$

Here and in what follows, we always assume $\alpha=6$ and denote by $\tilde{B}$ the smallest $\left(6, \beta_{6}\right)$-doubling ball of the form $6^{j} B$ with $j \in \mathbb{Z}_{+}$.

The following discrete coefficient $\tilde{K}_{B, S}^{(\kappa)}$ introduced by Bui and Duong [33] is very similar to the quantity $K_{B, S}$ which is introduced by Tolsa in [7].

Definition 4 [33]). For any $\kappa \in(1, \infty)$ and two balls $B, S \in \mathscr{X}$ satisfying $B \subset S$, define

$$
\tilde{K}_{B, S}^{(\kappa)}=1+\sum_{l=-\left\lfloor\log _{\kappa}\right\rfloor}^{N_{B, S}^{(\kappa)}} \frac{\mu\left(\kappa^{l} B\right)}{\lambda\left(c_{B}, \kappa^{l} r_{B}\right)},
$$

where $N_{B, S}^{(\kappa)}$ represents the smallest integer satisfying $\kappa^{N_{B, S}^{(\kappa)}} r_{B}$ $\geq r_{S}$.

Remark 5.

(i) By the definition of $N_{B, S}^{(\kappa)}$ and the fact $r_{B} \leq 2 r_{S}$, it is not difficult to get $N_{B, S}^{(\kappa)} \geq\left\lceil-\log _{\kappa} 2\right\rceil=-\left\lfloor\log _{\kappa} 2\right\rfloor$, which guarantees the definition of the $\tilde{K}_{B, S}^{(\kappa)}$ to make sense. Furthermore, Lin et al. [16] showed that, via a change of variables and (4), it is obvious to see that

$$
\tilde{K}_{B, S}^{(\kappa)} \sim 1+\sum_{l=1}^{N_{B, S}^{(\kappa)}+\left\lfloor\log _{\kappa} 2\right\rfloor+1} \frac{\mu\left(\kappa^{l} B\right)}{\lambda\left(c_{B}, \kappa^{l} r_{B}\right)}
$$

holds, where the implicit equivalent positive constants do not rely on the balls $B \subset S \subset \mathscr{X}$ but depend on the choice of $\kappa$ with $\kappa \in(1, \infty)$

(ii) Hytönen in [9] introduced a continuous version $K_{B, S}$ (also see [12]). That is, for any two balls $B, S \in \mathscr{X}$ satisfying $B \subset S$, set

$$
K_{B, S}=1+\int_{(2 S) \backslash B} \frac{1}{\lambda\left(c_{B}, d\left(x, c_{B}\right)\right)} \mathrm{d} \mu(x) .
$$

Via the simple computation, it is not difficult to see that $K_{B, S} \leq C \tilde{K}_{B, S}^{(\kappa)}$. Unfortunately, in general, $K_{B, S}$ and $\tilde{K}_{B, S}^{(\kappa)}$ are not equivalent, but, under the case of nondoubling measure, $K_{B, S} \sim \tilde{K}_{B, S}^{(\kappa)}$.

We now give out the definition of parameter $\theta$-type Marcinkiewicz integral $\mathscr{M}_{\theta}^{\rho}$. 
Definition 6. Let $\theta$ be a nonnegative, nondecreasing function on $(0, \infty)$ satisfying the following condition:

$$
\int_{0}^{1} \frac{\theta(t)}{t} \log \left(\frac{1}{t}\right) \mathrm{d} t<\infty
$$

Suppose that $K(\cdot, \cdot)$ is a locally integrable function defined on $\mathscr{X} \times \mathscr{X} \backslash\{(x, y): x=y\}$. Then, there exists a positive constant $C$ such that, for all $x, y \in \mathscr{X}$ with $x \neq y$

$$
|K(x, y)| \leq C \frac{d(x, y)}{\lambda(x, d(x, y))}
$$

and, for all $x, x^{\prime}, y$ with satisfying $d(x, y) \geq 2 d\left(x, x^{\prime}\right)$

$$
\begin{gathered}
\left|K(x, y)-K\left(x^{\prime}, y\right)\right|+\left|K(y, x)-K\left(y, x^{\prime}\right)\right| \\
\quad \leq C \theta\left(\frac{d\left(x, x^{\prime}\right)}{d(x, y)}\right) \frac{d(x, y)}{\lambda(x, d(x, y))} .
\end{gathered}
$$

Remark 7. Especially, if we take $\theta(t)=t^{\varepsilon}$ with $\varepsilon \in(0,1]$ as in (9), then the above kernel is just the standard kernel given in [20].

The parameter $\theta$-type Marcinkiewicz integral $\mathscr{M}_{\theta}^{\rho}$ associated with the above kernel $K$ satisfying (8) and (9) is defined by, for all $x \in \mathscr{X}$ and $\rho>0$

$$
M_{\theta}^{\rho}(f)(x)=\left(\int_{0}^{\infty}\left|\frac{1}{t^{\rho}} \int_{d(x, y) \leq t} \frac{K(x, y)}{[d(x, y)]^{1-\rho}} f(y) \mathrm{d} \mu(y)\right|^{2} \frac{\mathrm{d} t}{t}\right)^{\frac{1}{2}} .
$$

Remark 8.

(1) If we take $\theta(t)=t^{\varepsilon}$ with $\varepsilon \in(0,1]$ as in (9), and $\rho=1$ in (10), then the parameter $\theta$-type Marcinkiewicz integral $\mathscr{M}_{\theta}^{\rho}$ is just the Marcinkiewicz integral $\mathscr{M}$ on $(\mathscr{X}, d, \mu)$ given in [17]

(2) If we take $(\mathscr{X}, d, \mu)=\left(\mathbb{R}^{n},|\cdot|, \mu\right), \theta(t)=t^{\varepsilon}$, and $\rho \equiv 1$, then the parameter $\theta$-type Marcinkiewicz integral $\mathscr{M}_{\theta}^{\rho}$ is just the Marcinkiewicz integral $\mathscr{M}$ under nondoubling measure (see [34])

(3) If we take $(\mathscr{X}, d, \mu)=\left(\mathbb{R}^{n},|\cdot|, \mathrm{d} x\right), \theta(t)=t^{\varepsilon}, \rho \equiv 1$, and $K(x, y)=\Omega(x-y) /|x-y|^{n-1}$, then the parameter $\theta$-type Marcinkiewicz integral $\mathscr{M}_{\theta}^{\rho}$ is just the classical Marcinkiewicz integral $\mathscr{M}_{\Omega}$ introduced by Stein in [35] and its form as follows:

$$
\mathscr{M}_{\Omega}(f)(x)=\left(\int_{0}^{\infty}\left|\int_{d(x, y) \leq t} \frac{\Omega(x-y)}{|x-y|^{n-1}} f(y) \mathrm{d} y\right|^{2} \frac{\mathrm{d} t}{t^{3}}\right)^{1 / 2}, x \in \mathbb{R}^{n}
$$

(4) If we take $(\mathscr{X}, d, \mu)=\left(\mathbb{R}^{n},|\cdot|, \mathrm{d} x\right), \theta(t)=t^{\varepsilon}$, and $K(x$ , $y)=\Omega(x-y) /|x-y|^{n-1}$, then $\mathscr{M}_{\theta}^{\rho}$ defined in (10) is just the classical parameter Marcinkiewicz integral $\mathscr{M}_{\Omega}^{\rho}$ introduced by Hörmander in [36], that is

$\mathscr{M}_{\Omega}^{\rho}(f)(x)=\left(\int_{0}^{\infty}\left|\frac{1}{t^{\rho}} \int_{d(x, y) \leq t} \frac{\Omega(x-y)}{|x-y|^{n-\rho}} f(y) \mathrm{d} y\right|^{2} \frac{\mathrm{d} t}{t}\right)^{1 / 2}$

where $x \in \mathbb{R}^{n}$ and $\rho>0$.

Next, we recall the definition of $A_{p}^{\rho}(\omega)$ weight given in [14].

Definition 9. [14] Let $\rho \in[1, \infty)$ and $p \in(1, \infty)$. A nonnegative $\mu$-measurable function $\omega$ is called an $A_{p}^{\rho}(\mu)$ weight if there exists a positive constant $C$ such that, for all balls $B \subset$ $\mathscr{X}$

$$
\left(\frac{1}{\mu(\rho B)} \int_{B} \omega(x) \mathrm{d} \mu(x)\right)\left\{\frac{1}{\mu(\rho B)} \int_{B}[\omega(x)]^{1-p^{\prime}} \mathrm{d} \mu(x)\right\}^{p-1} \leq C .
$$

And a weight $\omega$ is called an $A_{1}^{\rho}(\mu)$ weight if there exists a positive constant $C$ such that, for all balls $B \subset \mathscr{X}$

$$
\frac{1}{\mu(\rho B)} \int_{B} \omega(x) \mathrm{d} \mu(x) \leq C \inf _{x \in B} \omega(x) .
$$

As in the classical setting, let $A_{\infty}^{\rho}(\mu):=\bigcup_{p=1}^{\infty} A_{p}^{\rho}(\mu)$.

The weighted generalized Morrey space $L^{p, \phi, \tau}(\omega)$ is defined as follows.

Definition 10. Let $\tau>1$ and $p \in(1, \infty)$ and $\omega$ be a weight. Suppose that $\phi:(0, \infty) \longrightarrow(0, \infty)$ is an increasing function. Then a weighted generalized Morrey space $L^{p, \phi, \tau}(\omega)$ is defined by

$$
L^{p, \phi, \tau}(\omega)=\left\{f \in L_{\mathrm{loc}}^{p}(\omega):\|f\|_{L^{p, \phi, \tau}(\omega)}<\infty\right\}
$$

where

$$
\|f\|_{L^{p, \phi, \tau}(\omega)}=\sup _{B}\left(\frac{1}{\phi(\omega(\tau B))} \int_{B}|f(x)|^{p} \omega(x) \mathrm{d} \mu(x)\right)^{1 / p} .
$$

We also denoted $W L^{p, \phi, \tau}(\omega)$ by the weighted weak generalized Morrey space of all locally integrable functions satisfying

$$
\begin{aligned}
\|f\|_{W L^{p, \phi, \tau}(\omega)}= & \sup _{B} \sup _{t>0} \frac{1}{[\phi(\omega(\tau B))]^{1 / p}} \\
& \cdot t \omega(\{x \in B:|f(x)|>t\})^{1 / p}<\infty .
\end{aligned}
$$


Remark 11. With an argument similar to that used in the proof of Lemma 2.3 and 2.4 in [14], it is not difficult to show that the norm of the weighted generalized Morrey space $\|\cdot\|_{L^{p, \phi, \tau}(\omega)}$ is independent of the choice of the parameter $\tau \in$ $(1, \infty)$.

The main results of this paper are stated as follows.

Theorem 12. Let $\tau, p \in[1, \infty), \rho \in[1, \infty), \omega \in A_{p}^{\rho}(\mu)$, and $\phi:(0, \infty) \longrightarrow(0, \infty)$ be an increasing function satisfying the following condition:

$$
\int_{m}^{\infty} \frac{\phi(z)}{z} \frac{d z}{z} \leq C \frac{\phi(m)}{m}, \text { for } 1<m<\infty
$$

Suppose that $\mathscr{M}_{\theta}^{\rho}$ defined by (10) associated with $K$ satisfying (8) and (9) is bounded on $L^{2}(\mu)$, and the mapping $t \mapsto \phi(t) / t$ is almost decreasing and there exists a positive constant $C$ such that

$$
\frac{\phi(t)}{t} \leq C \frac{\phi(s)}{s}
$$

for $s \geq t$. Then, $\mathscr{M}_{\theta}^{\rho}$ is bounded from $L^{p, \phi, \tau}(\omega) \cap L^{2}(\mu)$ into itself.

Theorem 13. Let $K$ satisfy (8) and (9), $\omega \in A_{p}^{\rho}(\mu)$, and $\phi$ : $(0, \infty) \longrightarrow(0, \infty)$ be an increasing function satisfying (18) and (19). Suppose that $\mathscr{M}_{\theta}^{\rho}$ defined in (10) is bounded on $L^{2}(\mu)$. Then, $M_{\theta}^{\rho}$ is bounded from $L^{p, \phi, \tau}(\omega)$ into $W L^{p, \phi, \tau}(\omega)$ for $\tau, p \in[1, \infty)$.

Finally, we make some conventions on notation. Throughout the paper, $C$ represents a positive constant which is independent of the main parameters but may be different from line to line. For a $\mu$-measurable set $E \subset \mathscr{X}, \chi_{E}$ denotes its characteristic function. For any $p \in[1, \infty]$, we denote by $p^{\prime}$ its conjugate index, that is $(1 / p)+\left(1 / p^{\prime}\right)=1$. For any ball $B, c_{B}$ and $r_{B}$ represent the center and radius of ball $B$, respectively. Furthermore, $m_{B}(f)$ denotes the mean value of the function $f$ over ball $B$, i.e., $m_{B}(f)=1 / \mu(B) \int_{B}$ $f(y) \mathrm{d} \mu(y)$.

\section{Proof of Main Theorems}

In this section, we will give out the proofs of Theorems 16 and 17. First, we need do recall the following lemmas.

We now recall the following properties of $A_{p}^{\rho}(\mu)$ weights from [15].

Lemma 14. [30] Let $\rho, p \in[1, \infty), \omega \in A_{p}^{\rho}(\omega)$ and $\eta \in[5 \rho, \infty)$. Then, there exist positive constants $C_{1}, C_{2} \in[1, \infty)$ such that (i) for any ball $B$ and $\mu$-measurable set $E \subset B$

$$
\frac{\omega(E)}{\omega(B)} \geq C_{2}^{-1}\left[\frac{\mu(E)}{\mu(\eta B)}\right]^{p}
$$

(ii) for any $\left(6, \beta_{6}\right)$-doubling ball $B$ and $\mu$-measurable set $E \subset B$

$$
\frac{\omega(E)}{\omega(B)} \leq 1-C_{1}^{-1}\left[1-\frac{\mu(E)}{\mu(B)}\right]^{\frac{1}{p}} .
$$

Finally, we recall the following lemma ensuring the integrability of functions [14].

Lemma 15. [27] Let $\psi:(0, \infty) \longrightarrow(0, \infty)$ be a function satisfying

$$
\int_{m}^{\infty} \psi(s) \frac{d s}{s} \leq C \psi(m), \text { for all } m>0
$$

Then there exists $\varepsilon>0$ such that $\int_{m}^{\infty} \psi(s) s^{\varepsilon}(d s / s) \leq C \psi$ $(m) m^{\varepsilon}$ for all $m>0$. In particular, for every $\eta \leq 1$, there exists a positive constant $C$ such that $\int_{m}^{\infty} \psi(s) s^{\eta}(d s / s) \leq C$ $\psi(m) m^{\eta}$ for all $m>0$.

The proofs of Theorems 16 and 17 are stated as follows.

Proof of Theorem 16. From Remark 11, we may assume that $\tau=6$ in (16). For a fixed doubling ball $B$, decompose $f(x)=$ $f_{1}(x)+f_{2}(x)$, where $f_{1}(x)=f(x) \chi_{6 B}(x)$. Write

$$
\|\mathscr{M}(f)\|_{L^{p, \phi, \tau}(\omega)} \leq\left\|\mathscr{M}\left(f_{1}\right)\right\|_{L^{p, \phi, \tau}(\omega)}+\left\|\mathscr{M}\left(f_{2}\right)\right\|_{L^{p, \phi, \tau}(\omega)}=: \mathrm{I}+\mathrm{II}
$$

In order to estimate $\mathrm{I}$, we first consider $\mathscr{M}\left(f_{1}\right)$. For any $x \in B$, by applying

$$
\begin{aligned}
\mathscr{M}_{\theta}^{\rho}\left(f_{1}\right)(x) \leq & \int_{X} \frac{|K(x, y)|}{[d(x, y)]^{1-\rho}}\left|f_{1}(y)\right|\left(\int_{d(x, y)}^{\infty} \frac{\mathrm{d} t}{t^{1+2 \rho}}\right)^{1 / 2} \mathrm{~d} \mu(y) \\
\leq & C \int_{6 B} \frac{|f(y)|}{\lambda(x, d(x, y))}[\omega(y)]^{1 / p}[\omega(y)]^{-1 / p} \mathrm{~d} \mu(y) \\
\leq & C\left(\int_{6 B} \frac{|f(y)|^{p} \omega(y)}{[\lambda(x, d(x, y))]^{p}} \mathrm{~d} \mu(y)\right)^{1 / p}\left[\frac{\mu(12 B)}{\omega(12 B)}\right]^{1 / p} \\
& \cdot[\mu(12 B)]^{1 / p^{\prime}} \times\left[\frac{1}{\mu(12 B)} \int_{6 B}(\omega(y))^{-p^{\prime} / p} \mathrm{~d} \mu(y)\right] \\
& \cdot\left(\frac{1}{\mu(12 B)} \int_{6 B} \omega(y) \mathrm{d} \mu(y)\right)^{1 / p}
\end{aligned}
$$




$$
\begin{aligned}
& \leq C\left(\int_{6 B} \frac{|f(y)|^{p} \omega(y)}{[\lambda(x, d(x, y))]^{p}} \mathrm{~d} \mu(y)\right)^{1 / p} \frac{\mu(12 B)}{[\omega(12 B)]^{1 / p}} \\
& \leq C\left(\sum_{j=0}^{\infty} \int_{6^{-j+1} B \backslash 6^{-j} B} \frac{|f(y)|^{p} \omega(y)}{[\lambda(x, d(x, y))]^{p}} \mathrm{~d} \mu(y)\right)^{1 / p} \frac{\mu(12 B)}{[\omega(12 B)]^{1 / p}} \\
& \leq C\|f\|_{L^{p, \phi, \tau}(\omega)} \sum_{j=0}^{\infty} \frac{\left[\phi\left(\omega\left(6^{-j+2} B\right)\right)\right]^{1 / p}}{\lambda\left(c_{B}, 6^{-j} r_{B}\right)} \frac{\mu\left(6^{-j} B\right)}{\mu\left(6^{-j} B\right)} \frac{\mu(12 B)}{[\omega(12 B)]^{1 / p}} \\
& \leq C\|f\|_{L^{p, \phi, \tau}(\omega)} \sum_{j=0}^{\infty} \frac{\left[\phi\left(\omega\left(6^{-j+2} B\right)\right)\right]^{1 / p}}{[\omega(12 B)]^{1 / p}} \frac{\mu(12 B)}{\mu\left(6^{-j} B\right)} \\
& \leq C\|f\|_{L^{p, \phi, \tau}(\omega)}\left[\frac{\phi(\omega(6 B))]^{1 / p},}{\omega(12 B)}\right]^{1 / p}
\end{aligned}
$$

and further, by applying (19) and Lemma 14, we can obtain that

$$
\begin{aligned}
\mathrm{I} & =\sup _{B}\left(\frac{1}{\phi(\omega(6 B))} \int_{B}\left|\mathscr{M}\left(f_{1}\right)(x)\right|^{p} \omega(x) \mathrm{d} \mu(x)\right)^{1 / p} \\
& \leq C\|f\|_{L^{p, \phi, \tau}(\omega)} \sup _{B}\left[\frac{\phi(\omega(6 B))}{\omega(12 B)}\right]^{1 / p}\left[\frac{\omega(B)}{\phi(\omega(6 B))}\right]^{1 / p} \\
& \leq C\|f\|_{L^{p, \phi, \tau}(\omega)} .
\end{aligned}
$$

Now we estimate II. For any $x \in B$, by applying (8), the Hölder inequality, and (13) and (16), we can get

$$
\begin{aligned}
\mathscr{M}_{\theta}^{\rho}\left(f_{2}\right)(x) \leq & C \sum_{j=1}^{\infty} \frac{1}{\lambda\left(c_{B}, 6^{j} r_{B}\right)} \int_{6^{j+1} B}|f(y)| \mathrm{d} \mu(y) \\
\leq & C \sum_{j=1}^{\infty} \frac{1}{\lambda\left(c_{B}, 6^{j} r_{B}\right)} \int_{6^{j+1} B}|f(y)|[\omega(y)]^{1 / p}[\omega(y)]^{-1 / p} \mathrm{~d} \mu(y) \\
\leq & C \sum_{j=1}^{\infty} \frac{1}{\lambda\left(c_{B}, 6^{j} r_{B}\right)}\left(\int_{6^{j+1} B}|f(y)|^{p} \omega(y) \mathrm{d} \mu(y)\right)^{1 / p} \\
& \times \frac{\mu\left(6^{j+2} B\right)}{\left[\omega\left(6^{j+1} B\right)\right]^{1 / p}}\left[\frac{1}{\mu\left(6^{j+1} B\right)} \int_{6^{j+1} B} \omega(y) \mathrm{d} \mu(y)\right]^{1 / p} \\
& \left.\times\left[\frac{1}{\mu\left(6^{j+1} B\right)}\right]_{6^{j+1} B}(\omega(y))^{-p^{\prime} / p} \mathrm{~d} \mu(y)\right]^{1 / p^{\prime}} \\
\leq & C \sum_{j=1}^{\infty} \frac{1}{\lambda\left(c_{B}, 6^{j} r_{B}\right)}\left(\int_{6^{j+1} B}|f(y)|^{p} \omega(y) \mathrm{d} \mu(y)\right)^{1 / p} \\
& \cdot \frac{\mu\left(6^{j+2} B\right)}{\left[\omega\left(6^{j+1} B\right)\right]^{1 / p}} \leq C\|f\|_{L^{p, \phi, \tau}(\omega)} \sum_{j=1}^{\infty} \\
& \cdot\left[\frac{\phi\left(\omega\left(6^{j+2} B\right)\right)}{\omega\left(6^{j+2} B\right)}\right]^{1 / p},
\end{aligned}
$$

and by the assumption (19) and Lemma 15, we have

$$
\sum_{j=1}^{\infty}\left[\frac{\phi\left(6^{j+1} \omega(6 B)\right)}{6^{j+1} \omega(6 B)}\right]^{1 / p} \leq C\left[\frac{\phi(\omega(6 B))}{\omega(6 B)}\right]^{1 / p}
$$

Thus, we have II $\leq C\|f\|_{L^{p, \phi, \tau}(\omega)}$. So the proof of Theorem 16 is completed.

Proof of Theorem 17. By applying the definition of $W L^{p, \phi, \tau}(\omega)$ and an argument similar to that used in the estimate of Theorem 12, it is not difficult to obtain that Theorem 13 holds. So, here we omit the detail.

\section{Data Availability}

The author confirms that no data were used to support this study.

\section{Conflicts of Interest}

The author declares that there is no conflict of interests regarding the publication of this paper.

\section{Acknowledgments}

This paper is supported by the Innovation Capacity Improvement Project for Colleges and Universities of Gansu Province (2020A-010), the Young Teachers' Scientific Research Ability Promotion Project of Northwest Normal University (NWNU-LKQN2020-07), and the Scientific Startup Foundation for Doctors of Northwest Normal University (0002020203).

\section{References}

[1] R. Coifman and G. Weiss, Analyse Harmonique Noncommutative sur certain Espaces Homogènes, vol. 242 of Lecture Notes in Mathematics, Springer-Verlag, Berlin-New York, 1971.

[2] R. R. Coifman and G. Weiss, "Extensions of Hardy spaces and their use in analysis," Bulletin of the American Mathematical Society, vol. 83, no. 4, pp. 569-646, 1977.

[3] V. Guliyev and Y. Sawano, "Linear and sublinear operators on generalized Morrey spaces with non-doubling measures," Publicationes Mathematicae Debrecen, vol. 83, no. 3, pp. 303-327, 2013.

[4] Y. Sawano, "Generalized Morrey spaces for non-doubling measures," NoDEA, vol. 15, no. 4-5, pp. 413-425, 2008.

[5] Y. Sawano and H. Tanaka, "Morrey spaces for non-doubling measures," Acta Mathematica Sinica, vol. 21, no. 6, pp. 1535-1544, 2005.

[6] Y. Sawano and H. Tanaka, "Sharp maximal inequalities and commutators on Morrey spaces with non-doubling measures," Taiwanese Journal of Mathematics, vol. 11, no. 4, pp. 10911112, 2007.

[7] X. Tolsa, "The space $H^{1}$ for nondoubling measures in terms of a grand maximal operator," Transactions of the American Mathematical Society, vol. 355, no. 1, pp. 315-348, 2003.

[8] X. Tolsa, "Littlewood-Paley theory and the T(1) theorem with non-doubling measures," Advances in Mathematics, vol. 164, no. 1 , pp. $57-116,2001$. 
[9] T. Hytönen, "A framework for non-homogeneous analysis on metric spaces, and the RBMO space of Tolsa," Publicationes Mathematiques, vol. 54, no. 2, pp. 485-504, 2010.

[10] C. Yonghui and Z. Jiang, "Morrey spaces for nonhomogeneous metric measure spaces," Abstract and Applied Analysis, vol. 2013, Article ID 196459, 8 pages, 2013.

[11] X. Fu, D. Yang, and D. Yang, “The molecular characterization of the Hardy space $H^{1}$ on non-homogeneous metric measure spaces and its application," Journal of Mathematical Analysis and Applications, vol. 410, no. 2, pp. 1028-1042, 2014.

[12] T. Hytönen, D. Yang, and D. Yang, "The Hardy space $H^{1}$ on non-homogeneous metric spaces," Mathematical Proceedings of the Cambridge Philosophical Society, vol. 153, no. 1, pp. 9$31,2012$.

[13] G. Lu and S. Tao, "Generalized Morrey spaces over nonhomogeneous metric measure spaces," Journal of the Australian Mathematical Society, vol. 103, no. 2, pp. 268-278, 2017.

[14] Y. Yan, J. Chen, and H. Lin, "Weighted Morrey spaces on nonhomogeneous metric measure spaces," Journal of Mathematical Analysis and Applications, vol. 452, no. 1, pp. 335-350, 2017.

[15] G. Hu, Y. Meng, and D. Yang, "Weighted norm inequalities for multilinear Calderón-Zygmund operators on nonhomogeneous metric measure spaces," Forum Mathematicum, vol. 26, no. 5, pp. 1289-1322, 2014.

[16] H. Lin, S. Wu, and D. Yang, "Boundedness of certain commutators over non-homogeneous metric measure spaces," Analysis and Mathematical Physics, vol. 7, no. 2, pp. 187218, 2017.

[17] H. Lin and D. Yang, "Equivalent boundedness of Marcinkiewicz integrals on non-homogeneous metric measure spaces," Science China Mathematics, vol. 57, no. 1, pp. 123-144, 2014.

[18] G. Lu, "Commutators of bilinear $\theta$-type Calderón-Zygmund operators on Morrey spaces over non-homogeneous spaces," Analysis Mathematica, vol. 46, no. 1, pp. 97-118, 2020.

[19] G. Lu and S. Tao, "Boundedness of commutators of Marcinkiewicz integrals on nonhomogeneous metric measure spaces," Journal of Function Spaces, vol. 2015, Article ID 548165, 12 pages, 2015.

[20] S. Tao and P. Wang, "Lipschitz estimate for Marcinkiewicz commutator on non-homogeneous metric measure spaces," Journal of Lanzhou University: Natural Sciences, vol. 52, no. 4, pp. 530-534, 2016.

[21] C. Morrey, "On the solutions of quasi-linear elliptic partial differential equations," Transactions of the American Mathematical Society, vol. 43, no. 1, pp. 126-166, 1938.

[22] V. Guliyev, "Integral operators on function spaces on the homogeneous groups and on domains in $\mathbb{R}^{n}$, Doctor's degree dissertation," Mat. Inst. Steklov, Moscow, 1994, In Russian.

[23] T. Mizuhara, "Boundedness of some classical operators on generalized Morrey spaces, Harmonic analysis," ICM 90 Satellite Proceedings, S. Igari, Ed., , pp. 183-189, Springer-Verlag, Tokyo, 1991.

[24] E. Nakai, "Hardy-Littlewood maximal operator, singular integral operators and the Riesz potentials on generalized Morrey spaces," Mathematische Nachrichten, vol. 166, no. 1, pp. 95103, 1994.

[25] V. Guliyev, Function Spaces, Integral Operators and Two Weighted Inequalities on Homogeneous Groups, Some Applications, Casioglu, Baku, 1999, In Russian.
[26] V. Guliyev, "Boundedness of the maximal, potential and Singular operators in the generalized Morrey spaces," Journal of Inequalities and Applications, vol. 2009, no. 1, Article ID 503948, 2009.

[27] Y. Komori and S. Shirai, "Weighted Morrey spaces and a singular integral operator," Mathematische Nachrichten, vol. 282, no. 2, pp. 219-231, 2009.

[28] S. Nakamura and Y. Sawano, "The singular integral operator and its commutator on weighted Morrey spaces," Collectanea Mathematica, vol. 68, no. 2, pp. 145-174, 2017.

[29] V. Guliyev, "Generalized weighted Morrey spaces and higher order commutators of sublinear operators," Eurasian Mathematical Journal, vol. 3, no. 3, pp. 33-61, 2012.

[30] V. S. Guliyev and F. C. Alizadeh, "Multilinear commutators of Calderón-Zygmund operator on generalized weighted Morrey spaces," Journal of Function Spaces, vol. 2014, Article ID 710542, 9 pages, 2014.

[31] T. Karaman, V. S. Guliyev, and A. Serbrtci, "Boundedness of sublinear operators generated by Calderón-Zygmund operators on generalized weighted Morrey spaces," Annals of the Alexandru Ioan Cuza University - Mathematics, vol. 60, no. 1, article 227"C244, 2014.

[32] S. Nakamura, "Generalized weighted Morrey spaces and classical operators," Mathematische Nachrichten, vol. 289, no. 17-18, pp. 2235-2262, 2016.

[33] T. A. Bui and X. T. Duong, "Hardy spaces, regularized BMO spaces and the boundedness of Calderón-Zygmund operators on non-homogeneous spaces," Journal of Geometric Analysis, vol. 23, no. 2, pp. 895-932, 2013.

[34] G. Hu, H. Lin, and D. Yang, "Marcinkiewicz integrals with non-doubling measures," Integral Equations and Operator Theory, vol. 58, no. 2, pp. 205-238, 2007.

[35] E. Stein, "On the functions of Littlewood-Paley, Lusin, and Marcinkiewicz," Transactions of the American Mathematical Society, vol. 88, no. 2, pp. 430-466, 1958.

[36] L. Hörmander, "Estimates for translation invariant operators in $L^{p}$ spaces," Acta Mathematica, vol. 104, no. 1-2, pp. 93140, 1960. 